\title{
Performance Analysis of Resampling Techniques on Class Imbalance Issue in Software Defect Prediction
}

\author{
Ahmed Iqbal, Shabib Aftab, Faseeha Matloob \\ Department of Computer Science, Virtual University of Pakistan \\ E-mail: ahmedeqbal@gmail.com, shabib.aftab@gmail.com, faseeham7@gmail.com
}

Received: 17 August 2019; Accepted: 14 September 2019; Published: 08 November 2019

\begin{abstract}
Absract-Predicting the defects at early stage of software development life cycle can improve the quality of end product at lower cost. Machine learning techniques have been proved to be an effective way for software defect prediction however an imbalance dataset of software defects is the main issue of lower and biased performance of classifiers. This issue can be resolved by applying the re-sampling methods on software defect dataset before the classification process. This research analyzes the performance of three widely used resampling techniques on class imbalance issue for software defect prediction. The resampling techniques include: "Random Under Sampling", "Random Over Sampling" and "Synthetic Minority Oversampling Technique (SMOTE)". For experiments, 12 publically available cleaned NASA MDP datasets are used with 10 widely used supervised machine learning classifiers. The performance is evaluated through various measures including: F-measure, Accuracy, MCC and ROC. According to results, most of the classifiers performed better with "Random Over Sampling" technique in many datasets.
\end{abstract}

Index Terms-Software Defect predication, Imbalanced Dataset, Resampling Methods, Random Under Sampling, Random Oversampling, Synthetic Minority Oversampling Technique.

\section{INTRODUCTION}

Developers and researchers have always been concerned to develop high quality softwares at lower cost $[7,8,9]$. Predicting the defects at early stage of development life cycle can achieve this goal as the cost of fixing the defects increases exponently at later stages [8], [20,21]. Software defect prediction is the problem of binary classification in which we have to classify the particular module as defective or non-defective $[7,8,9]$. Supervised machine learning techniques have been widely used to solve the binary classification problems such as Sentiment Analysis [12, 13, 14, 15], Rainfall Prediction [16, 17], and DoS attack detection [18, 19]. The supervised machine learning techniques uses preclassified data (training data) in order to train the classifier. During the training process, classification rules are developed which are used to classify the unseen data (test data) $[10,11]$. The datasets of software defects are usually skewed in which too many instances are related to one class and very less instances belong to second class. For instance, normally less records are related to defective class and too much records are related to nondefective class. The class with less instances is known as minority class and the class with the too many instances is known as the majority class. The imbalance between these two classes is reflected by "Imbalance ratio", which is the ratio of the number of instances in majority class to that of a minority class. [1]. In software defect datasets, the instances related to non-defective class are usually high with respect to defective instances as shown in this table 1. Therefore the particular classifiers trained on such imbalanced datasets may produce the bias result and may classify the minority instance as majority instance. To resolve this issue, many techniques are available. This paper uses three well known resampling techniques to handle the class imbalance issue for software defect prediction. The resampling techniques include: Random Under Sampling (ROS), Random Over Sampling (RUS) and Synthetic Minority Oversampling Technique (SMOTE). Twelve (12) publically available cleaned NASA MDP datasets are used for experiments along with 10 widely used supervised machine learning classifiers including: "Naïve Bayes (NB), Multi-Layer Perceptron (MLP). Radial Basis Function (RBF), Support Vector Machine (SVM), K Nearest Neighbor (KNN), $\mathrm{kStar}\left(\mathrm{K}^{*}\right)$, One Rule (OneR), PART, Decision Tree (DT), and Random Forest (RF)". Performance evaluation is performed by using: "F-measure, Accuracy, MCC and ROC". The results reflects that ROS performed better in almost all the datasets.

The rest of the paper is organized as follows: Section 2 discusses the related work done on Class Imbalance issue. Section 3 elaborates the materials and methods which are used in this research. Section 4 reflects the results of experiments. Section 5 finally concludes this research.

\section{RELATED WORK}

Many researchers have worked to resolve the class imbalance issue since the last decade. Some of the related studies are discussed here. In [1], the authors proposed SOS-BUS which is a hybrid resampling technique. This approach integrated a well- known oversampling technique SMOTE with the newly developed Under Sampling Technique. The proposed approach focusses on the necessary data of majority class and avoid the 
removal stage from random under-sampling. The results reflected that the proposed technique performed better in Area under ROC Curve (AUC). In [2], the researchers discussed class imbalance learning methods and elaborated that how these methods can be used for effective software defect prediction. They investigated various class imbalance learning methods such as resampling techniques, threshold moving techniques, and ensemble algorithms. AdaBoost.NC reflected high performance in Balance, G-mean, and Area Under the Curve (AUC). Moreover an improvement in AdaBoost.NC has also been proposed in which parameter adjustment is performed automatically during the training process. The proposed version proved to be more effective and efficient. Researchers in [3] discussed two resampling techniques which are the extensions of SMOTE and RUS. They have studied and discussed an ensemble learning method AdaBoost.R2 to resolve the data imbalance issue for software defect prediction. The researchers discussed that the extensions of SMOTE and RUS, SmoteND and RusND are the effective techniques to resolve the imbalance issue in software defect datasets. Experiments on 6 datasets with two performance measures have showed the effectives of these techniques. Moreover in order to improve the performance of these techniques, AdaBoost.R2 algorithm is integrated and made these techniques as SmoteNDBoost and RusNDBoost. Experiments reflected that the hybrid approach outperformed the individual techniques including SmoteND, RusND and AdaBoost.R2). Researchers in [4] analyzed the performance of SMOTE on the issue of class imbalance on software defect prediction. They have analyzed that at which extent the SMOTE technique can improve the classification of defective software modules. The researchers reflected that after applying the SMOTE technique the dataset became more balanced and more accurate results were noted on four benchmark datasets. In [5], the researchers have proposed a software defect prediction system named "Weighted Least Squares Twin Support Vector Machine" (WLSTSVM). The proposed system works by assigning the higher misclassification cost to the instances related to defective class and lower cost to the instances related to non-defective class. Experiments were performed by using 8 software defect datasets which showed that the proposed system performed better in non-parametric Wilcoxon signed rank test. Researchers in [6] used three re sampling techniques on credit card dataset in order to overcome the class imbalance issue. The techniques include: Random Under Sampling, Random Over Sampling and Synthetic Minority Oversampling Technique. The performance was evaluated by using Sensitivity, Specificity, Accuracy, Precision, Area Under Curve (AUC) and Error Rate. The results reflected that the resampled datasets brought better performance. Researchers in [7] analyzed the performance of various widely used supervised classifiers on software defect prediction. For experiments, 12 publically available NASA datasets were used. The classification techniques included: "Naïve Bayes (NB), Multi-Layer Perceptron
(MLP). Radial Basis Function (RBF), Support Vector Machine (SVM), K Nearest Neighbor (KNN), kStar (K*), One Rule (OneR), PART, Decision Tree (DT), and Random Forest (RF)". The performance is evaluated by using Precision, Recall, F-Measure, Accuracy, MCC, and ROC Area. The researchers have observed that that neither the Accuracy and nor the ROC can be used as an effective performance measure as both of these did not react on class imbalance issue. However, Precision, Recall, F-Measure and MCC reacted to class imbalance problem in the results.

\section{MATERIALS AND METHODS}

This research aims to analyze the performance of three well known resampling techniques on class imbalance issue for software defect prediction. For this purpose a comparison framework (Fig. 1) is used in which performance of each re-sampling technique is analyzed with various well known classifiers. The framework consists of four stages: 1) Dataset selection, 2) Resampling, 3) Classification, and 4) reflection and analysis of Results. First stage deals with the selection of datasets in which 12 cleaned NASA MDP datasets are used for experiments including: "CM1, JM1, KC1, KC3, MC1, MC2, MW1, PC1, PC2, PC3, PC4 and PC5 (Table I)". Each of the used dataset represents a particular NASA's software system. The datasets include various quality metrics in the form of attributes along with known output class. The output class is also called the target class which is predicted on behalf of other attributes. The attribute which holds the target/output class is known as dependent attribute and other attributes are known as independent attributes as those are used to predict the dependent attribute.

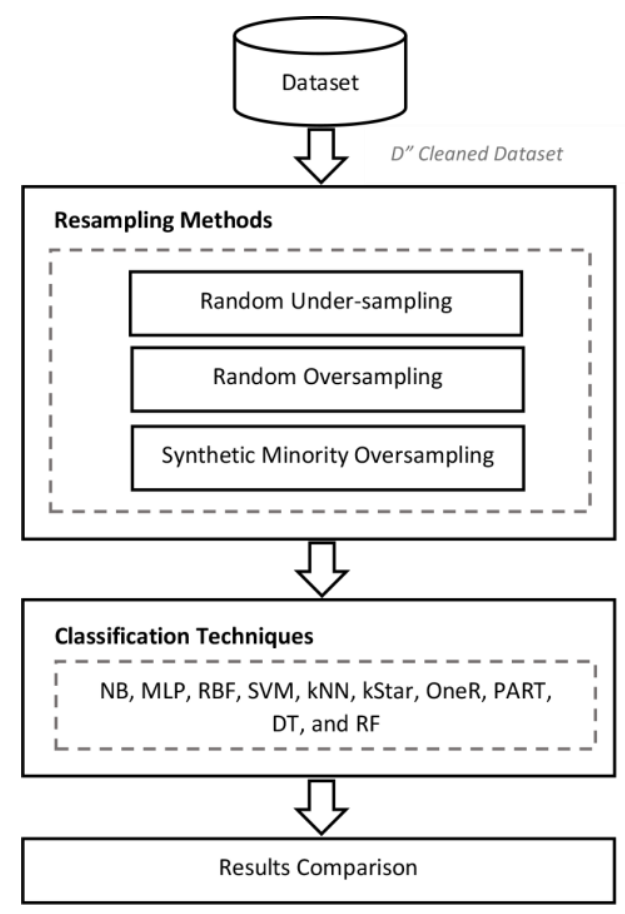

Fig.1. Comparison Framework 
Table 1. Cleaned NASA Software Datasets [22], [23]

\begin{tabular}{|c|c|c|c|c|}
\hline Dataset & Attributes & Modules & Defective & $\begin{array}{c}\text { Non- } \\
\text { Defective }\end{array}$ \\
\hline CM1 & 38 & 327 & 42 & 285 \\
\hline JM1 & 22 & 7,720 & 1,612 & 6,108 \\
\hline KC1 & 22 & 1,162 & 294 & 868 \\
\hline KC3 & 40 & 194 & 36 & 158 \\
\hline MC1 & 39 & 1952 & 36 & 1916 \\
\hline MC2 & 40 & 124 & 44 & 80 \\
\hline MW1 & 38 & 250 & 25 & 225 \\
\hline PC1 & 38 & 679 & 55 & 624 \\
\hline PC2 & 37 & 722 & 16 & 706 \\
\hline PC3 & 38 & 1,053 & 130 & 923 \\
\hline PC4 & 38 & 1,270 & 176 & 1094 \\
\hline PC5 & 39 & 1694 & 458 & 1236 \\
\hline
\end{tabular}

values of either "Y" or "N". "Y" means that particular instance (which represents a module) is defective and "N" reflects that it is non-defective. Two versions of NASA MDP cleaned datasets are provided by [22], D' and D". D' includes the duplicate and inconsistent instances whereas D" do not include duplicate and inconsistent instances. We have used D" version of cleaned datasets (Table 1) available at [23]. This version of cleaned dataset is already used and discussed by $[7,8,9]$, $[24,25,26]$. Table 2 reflects the cleaning criteria implemented by [22]. Table 1 reflects some crucial information about the datasets which are used in this research such as: Dataset name, No of attributes each dataset contains, No of modules (instances) in each dataset, No of defective modules and No of non-defective modules.

The dependent attribute in the used datasets have the

Table 2. Cleaning Criteria Applied to Noisy NASA Datasets [22], [24]

\begin{tabular}{|c|c|l|}
\hline Criterion & Data Quality Category & \multicolumn{1}{c|}{ Explanation } \\
\hline 1. & Identical cases & $\begin{array}{l}\text { "Instances that have identical values for all metrics } \\
\text { including class label". }\end{array}$ \\
\hline 2. & Inconsistent cases & $\begin{array}{l}\text { "Instances that satisfy all conditions of Case 1, but where } \\
\text { class labels differ". }\end{array}$ \\
\hline 3. & Cases with missing values & "Instances that contain one or more missing observations". \\
\hline 4. & Cases with conflicting feature values & $\begin{array}{l}\text { "Instances that have 2 or more metric values that violate } \\
\text { some referential integrity constraint. For example, LOC } \\
\text { TOTAL is less than Commented LOC. However, } \\
\text { Commented LOC is a subset of LOC TOTAL". }\end{array}$ \\
\hline 5. & Cases with implausible values & $\begin{array}{l}\text { "Instances that violate some integrity constraint. For } \\
\text { example, value of LOC=1.1" }\end{array}$ \\
\hline
\end{tabular}

Table 3. Comparison of Resampling Methods [6]

\begin{tabular}{|c|l|l|l|}
\hline & \multicolumn{1}{|c|}{ RUS } & \multicolumn{1}{|c|}{ ROS } & \multicolumn{1}{|c|}{ SMOTE } \\
\hline Process & $\begin{array}{l}\text { Removes the instances of } \\
\text { majority class randomly and } \\
\text { reduces the dataset. }\end{array}$ & $\begin{array}{l}\text { Increases the instances of } \\
\text { minority class by duplicating } \\
\text { randomly }\end{array}$ & $\begin{array}{l}\text { Increases the instances of minority } \\
\text { class by extrapolating between } \\
\text { preexisting minority } \\
\text { instances } \\
\text { which obtained by KNN. }\end{array}$ \\
\hline Strength & Shorter convergence time. & $\begin{array}{l}\text { No information is lost and } \\
\text { can improve the performance } \\
\text { produce better results. }\end{array}$ & $\begin{array}{l}\text { Effective in improving the } \\
\text { classification accuracy of the } \\
\text { minority data. }\end{array}$ \\
\hline Limitation & $\begin{array}{l}\text { Important information is lost } \\
\text { due to the shrinkage of } \\
\text { majority class }\end{array}$ & $\begin{array}{l}\text { Overfitting issue due to } \\
\text { multiple tied instances. } \\
\text { spread on both minority and } \\
\text { majority data, hence reduced the } \\
\text { performance of classification. }\end{array}$ \\
\hline
\end{tabular}

Second stage uses 3 widely used re sampling techniques (Table 3 ) to resolve the class imbalance issue in datasets. The resampling techniques include: RUS (Random Under-Sampling), ROS (Random OverSampling), SMOTE (Synthetic Minority Over-sampling Technique) which are discussed below:

\section{A. RUS (Random Under-Sampling)}

This technique reduces the imbalance ratio in dataset by removing some of the instances of majority class and makes the classes equal in terms of related instances. The balanced dataset may lose the important information during the removal of instances from majority class due to which the classifier may reflect worse performance.

\section{B. ROS (Random Over-Sampling)}

This technique reduces the imbalance ratio in dataset by duplicating the instances in minority class. With this approach the existing instances retains in the dataset but the volume of the dataset increases due to duplication. Both the classes can get balance with this technique however over-fitting problem can occur which can degrade the performance of classifier.

\section{SMOTE (Synthetic Minority Over-sampling Technique)}

Synthetic Minority Over-sampling Technique (SMOTE) is the widely used sampling techniques which creates additional synthetic instances in the minority 
class instead of duplication. The steps of this technique are discussed below:

1. A random number is generated between 0 and 1 .

2. The difference between the feature vector of minority class sample and its nearest neighbor is calculated.

3. The difference calculated in step 2 is multiplied with the random number generated in step 1.

4. The result achieved in step 3 is added to the feature vector of minority class sample.

5 . The result of step 4 identifies the newly generated sample.

Third stage deals with the classification by using various widely used supervised learning techniques including: Naïve Bayes (NB), Multi-Layer Perceptron (MLP). Radial Basis Function (RBF), Support Vector Machine (SVM), K Nearest Neighbor (KNN), kStar (K*), One Rule (OneR), PART, Decision Tree (DT), and Random Forest (RF).

Fourth and final stage of the comparison framework deals with the extraction and reflection of results and is discussed in next section (Section 4).

\section{RESULTS AND DISCUSSION}

The datasets after re sampling are given to classification techniques as input with $70: 30$ ratio (70 \% training data and $30 \%$ test data). Performance is evaluated through various measures generated from confusion matrix including: Precision, Recall, F-measure, Accuracy, MCC and ROC.

The confusion matrix is shown in (Fig. 2) and consists of following parameters [7].

True Positive (TP): "Instances which are actually positive and also classified as positive".

False Positive (FP): "Instances which are actually negative but classified as positive".
False Negative (FN): "Instances which are actually positive but classified as negative".

True Negative (TN): "Instances which are actually negative and also classified as negative".

Performance measures are based on the parameters of confusion matrix and are discussed below $[7,8,9]$.

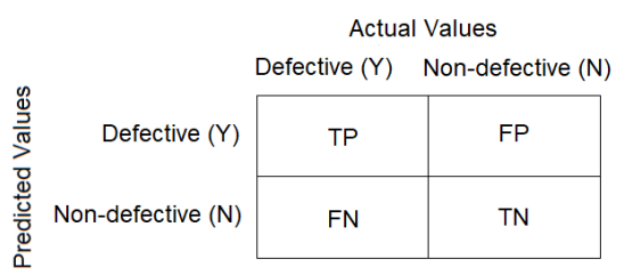

Fig.2. Confusion Matrix

$$
\begin{gathered}
\text { Precision }=\frac{T P}{(T P+F P)} \\
\text { Recall }=\frac{T P}{(T P+F N)}
\end{gathered}
$$

$$
F-\text { measure }=\frac{\text { Precision }^{*} \text { Recall }^{*} 2}{(\text { Precision }+ \text { Recall })}
$$

$$
\begin{gathered}
\text { Accuracy }=\frac{T P+T N}{T P+T N+F P+F N} \\
A U C=\frac{1+T P_{r}-F P_{r}}{2}
\end{gathered}
$$

\begin{tabular}{|c|c|c|c|c|c|c|c|c|c|c|c|c|c|c|c|c|}
\hline \multirow[b]{2}{*}{ Classifiers } & \multicolumn{4}{|c|}{ F-Measure } & \multicolumn{4}{|c|}{ Accuracy } & \multicolumn{4}{|c|}{ ROC Area } & \multicolumn{4}{|c|}{ MCC } \\
\hline & No Tec & RUS & ROS & SMOTE & No Tec & RUS & ROS & SMOTE & $\begin{array}{l}\text { No } \\
\text { Tec }\end{array}$ & RUS & ROS & SMOTE & $\begin{array}{l}\text { No } \\
\text { Tec }\end{array}$ & RUS & ROS & SMOTE \\
\hline NB & & 0.316 & & & 82.653 & 48.000 & 59.183 & & 0.703 & 0.535 & 0.685 & 0.806 & 0.097 & -0.06 & .217 & 0.308 \\
\hline MLP & & & 807 & & 6.734 & 64.000 & 89.795 & & 0.634 & 0.622 & 0.923 & & -0.060 & 0.316 & 0.813 & 0.138 \\
\hline RBF & $?$ & 0.667 & 0.736 & 71 & 90.816 & 60.000 & 71.428 & 73.873 & 0.702 & 0.532 & 0.741 & 0.686 & $?$ & 0.243 & 434 & .161 \\
\hline SVM & $?$ & 0.667 & 0.684 & 0.065 & 90.816 & 60.000 & 63.265 & 73.873 & 0.500 & 0.609 & 0.633 & 0.517 & $?$ & 0.243 & 0.281 & 0.157 \\
\hline kNN & 0.083 & 0.667 & 0.867 & 0.517 & 77.551 & 60.000 & \begin{tabular}{|l|}
84.693 \\
\end{tabular} & 74.774 & 0.477 & 0.609 & 0.855 & 0.670 & -0.037 & 0.243 & 0.729 & 0.347 \\
\hline kStar & 083 & 0.348 & 899 & 571 & 7.551 & 40.000 & \begin{tabular}{|l|}
88.775 \\
\end{tabular} & 78.378 & 0.538 & 0.417 & 0.946 & 0.761 & -0.037 & -0.20 & .796 & 9.430 \\
\hline OneR & 0.000 & 0.667 & 0.759 & 0.273 & 85.714 & 60.000 & 73.469 & 71.171 & 0.472 & 0.609 & 0.735 & 0.551 & -0.074 & 0.243 & 0.479 & 0.135 \\
\hline PART & $?$ & 0.444 & 0.913 & 0.600 & 90.816 & 40.000 & 90.816 & 78.378 & 0.610 & 0.413 & 0.912 & 0.684 & $?$ & -0.19 & 0.821 & 0.452 \\
\hline DT & 0.154 & 0.581 & 0.846 & 0.458 & 77.551 & 48.000 & \begin{tabular}{|l|}
83.673 \\
\end{tabular} & 76.576 & 0.378 & 0.439 & 0.850 & 0.595 & 0.041 & -0.02 & 0.679 & 0.338 \\
\hline $\mathbf{R F}$ & 0.000 & 0.571 & 0.899 & 0.462 & 89.795 & 52.000 & 88.775 & 81.081 & 0.761 & 0.465 & 0.991 & 0.924 & -0.032 & 0.053 & 0.796 & 0.488 \\
\hline
\end{tabular}

$$
M C C=\frac{T N * T P-F N * F P}{\sqrt{(F P+T P)(F N+T P)(T N+F P)(T N+F N)}}
$$

Table 4. CM1 Results 
Table 5. JM1 Results

\begin{tabular}{|c|c|c|c|c|c|c|c|c|c|c|c|c|c|c|c|c|}
\hline \multirow[b]{2}{*}{ Classifiers } & \multicolumn{4}{|c|}{ F-Measure } & \multicolumn{4}{|c|}{ Accuracy } & \multicolumn{4}{|c|}{ ROC Area } & \multicolumn{4}{|c|}{ MCC } \\
\hline & No Tec & RUS & ROS & SMOTE & No Tec & RUS & ROS & SMOTE & No Tec & RUS & ROS & SMOTE & $\begin{array}{l}\text { No } \\
\text { Tec }\end{array}$ & RUS & ROS & SMOTE \\
\hline NB & 0.318 & 0.307 & 0.310 & 0.290 & 79.835 & 57.600 & 56.217 & 68.035 & 0.663 & 0.650 & 0.652 & 0.629 & 0.251 & 0.210 & 0.199 & 0.188 \\
\hline MLP & 0.146 & 0.639 & 0.525 & 0.483 & 80.354 & 59.152 & 61.312 & 67.750 & 0.702 & 0.671 & 0.673 & 0.666 & 0.206 & 0.197 & 0.250 & 0.253 \\
\hline RBF & 0.181 & 0.601 & 0.605 & 0.403 & 80.397 & 62.771 & 63.385 & 69.000 & 0.713 & 0.667 & 0.675 & 0.675 & 0.215 & 0.225 & 0.273 & 0.241 \\
\hline SVM & $?$ & 0.497 & 0.504 & 0.217 & 79.188 & 62.771 & 61.053 & 67.750 & 0.500 & 0.623 & 0.613 & 0.545 & $?$ & 0.284 & 0.252 & 0.167 \\
\hline kNN & 0.348 & 0.594 & 0.867 & 0.565 & 73.963 & 59.565 & 85.751 & 71.035 & 0.591 & 0.596 & 0.850 & 0.672 & 0.186 & 0.192 & 0.720 & 0.348 \\
\hline kStar & 0.355 & 0.567 & 0.869 & 0.646 & 75.993 & 59.462 & 85.794 & 76.071 & 0.572 & 0.634 & 0.934 & 0.794 & 0.212 & 0.188 & 0.723 & 0.465 \\
\hline OneR & 0.216 & 0.560 & 0.672 & 0.607 & 77.158 & 56.256 & 67.055 & 77.678 & 0.543 & 0.563 & 0.671 & 0.711 & 0.126 & 0.125 & 0.341 & 0.478 \\
\hline PART & 0.037 & 0.678 & 0.658 & 0.606 & 79.490 & 65.563 & 66.968 & 76.142 & 0.714 & 0.697 & 0.738 & 0.786 & 0.104 & 0.319 & 0.342 & 0.446 \\
\hline DT & 0.348 & 0.619 & 0.834 & 0.623 & 79.101 & 63.909 & 82.728 & 76.821 & 0.671 & 0.654 & 0.855 & 0.777 & 0.252 & 0.278 & 0.655 & 0.464 \\
\hline $\mathbf{R F}$ & 0.284 & 0.671 & 0.885 & 0.690 & 80.181 & 66.597 & 87.953 & 81.071 & 0.738 & 0.715 & 0.960 & 0.840 & 0.244 & 0.334 & 0.761 & 0.564 \\
\hline
\end{tabular}

Table 6. KC1 Results

\begin{tabular}{|c|c|c|c|c|c|c|c|c|c|c|c|c|c|c|c|c|}
\hline \multirow{2}{*}{ Classifiers } & \multicolumn{4}{|c|}{ F-Measure } & \multicolumn{4}{|c|}{ Accuracy } & \multicolumn{4}{|c|}{ ROC Area } & \multicolumn{4}{|c|}{ MCC } \\
\hline & No Tec & RUS & ROS & SMOTE & No Tec & RUS & ROS & SMOTE & $\begin{array}{l}\text { No } \\
\text { Tec }\end{array}$ & RUS & ROS & SMOTE & $\begin{array}{l}\text { No } \\
\text { Tec }\end{array}$ & RUS & ROS & SMOTE \\
\hline NB & 0.400 & 0.516 & 0.498 & 0.468 & 74.212 & 64.772 & 61.318 & 66.132 & 0.694 & 0.719 & 0.669 & 0.692 & 0.250 & 0.300 & 0.253 & 0.280 \\
\hline MLP & 0.358 & 0.504 & 0.692 & 0.480 & 77.363 & 67.613 & 69.627 & 67.734 & 0.736 & 0.755 & 0.744 & 0.714 & 0.296 & 0.398 & 0.393 & 0.322 \\
\hline RBF & 0.362 & 0.649 & 0.648 & 0.512 & 78.796 & 69.318 & 67.335 & 67.276 & 0.713 & 0.762 & 0.705 & 0.702 & 0.347 & 0.381 & 0.350 & 0.307 \\
\hline SVM & 0.085 & 0.639 & 0.611 & 0.445 & 75.358 & 70.454 & 63.896 & 67.505 & 0.521 & 0.695 & 0.639 & 0.623 & 0.151 & 0.408 & 0.280 & 0.324 \\
\hline kNN & 0.395 & 0.584 & 0.858 & 0.684 & 69.341 & 61.931 & 85.673 & 72.997 & 0.595 & 0.616 & 0.868 & 0.728 & 0.190 & 0.233 & 0.714 & 0.449 \\
\hline kStar & 0.419 & 0.650 & 0.836 & 0.717 & 72.206 & 68.750 & 83.094 & 76.201 & 0.651 & 0.686 & 0.912 & 0.843 & 0.238 & 0.370 & 0.663 & 0.512 \\
\hline OneR & 0.256 & 0.656 & 0.661 & 0.671 & 73.352 & 64.204 & 65.616 & 77.345 & 0.551 & 0.648 & 0.656 & 0.742 & 0.147 & 0.298 & 0.313 & 0.536 \\
\hline PART & 0.255 & 0.664 & 0.671 & 0.475 & 76.504 & 55.113 & 72.779 & 69.107 & 0.636 & 0.680 & 0.813 & 0.727 & 0.239 & 0.227 & 0.484 & 0.366 \\
\hline DT & 0.430 & 0.500 & 0.798 & 0.679 & 75.644 & 64.772 & 79.369 & 72.082 & 0.606 & 0.664 & 0.784 & 0.740 & 0.291 & 0.305 & 0.588 & 0.434 \\
\hline $\mathbf{R F}$ & 0.454 & 0.620 & 0.861 & 0.790 & 77.937 & 63.068 & 85.959 & 82.837 & 0.751 & 0.733 & 0.935 & 0.890 & 0.346 & 0.263 & 0.720 & 0.645 \\
\hline
\end{tabular}

Table 7. KC3 Results

\begin{tabular}{|c|c|c|c|c|c|c|c|c|c|c|c|c|c|c|c|c|}
\hline \multirow{2}{*}{ Classifiers } & \multicolumn{4}{|c|}{ F-Measure } & \multicolumn{4}{|c|}{ Accuracy } & \multicolumn{4}{|c|}{ ROC Area } & \multicolumn{4}{|c|}{ MCC } \\
\hline & No Tec & RUS & ROS & SMOTE & No Tec & RUS & ROS & SMOTE & $\begin{array}{l}\text { No } \\
\text { Tec }\end{array}$ & RUS & ROS & SMOTE & $\begin{array}{l}\text { No } \\
\text { Tec }\end{array}$ & RUS & ROS & SMOTE \\
\hline NB & 0.421 & 0.526 & 0.588 & 471 & 81.034 & 59.090 & 3.793 & 3.913 & 0.769 & 0.846 & 0.712 & 0.686 & 0.309 & 0.302 & 0.358 & 0.364 \\
\hline MLP & 0.375 & 0.667 & 0.857 & 0.634 & 82.758 & 63.636 & 84.482 & 78.260 & 0.733 & 0.761 & 0.851 & 0.824 & 0.295 & 0.277 & 0.692 & 0.490 \\
\hline RBF & 0.000 & 0.526 & 0.733 & 0.467 & 77.586 & 59.090 & 72.413 & 76.811 & 0.735 & 0.829 & 0.772 & 0.724 & -0.107 & 0.302 & 0.463 & 0.475 \\
\hline SVM & ? & 0.526 & 0.667 & 0.160 & 82.758 & 59.090 & 67.241 & 69.565 & 0.500 & 0.637 & 0.688 & $0.0+3$ & $?$ & 0.302 & 0.378 & $24+4$ \\
\hline kNN & 0.364 & 0.600 & 0.889 & 0.600 & 75.862 & 63.636 & 87.931 & 76.811 & 0.617 & 0.675 & 0.093 & 0.101 & 0.218 & 0.370 & 0.762 & 0.452 \\
\hline kStar & 0.300 & 0.609 & 0.923 & 0.667 & 75.862 & 59.090 & 91.379 & 79.710 & 0.528 & 0.521 & 0.942 & 0.818 & 0.154 & 0.203 & 0.826 & 0.528 \\
\hline OneR & 0.375 & 0.476 & 0.836 & 0.439 & 82.758 & 50.000 & 81.034 & 66.666 & 0.619 & 0.526 & 0.804 & 0.598 & 0.295 & 0.052 & 0.612 & 0.210 \\
\hline PART & 0.143 & 0.640 & 0.866 & 0.683 & 79.3103 & 59.090 & 84.482 & 81.159 & 0.788 & 0.598 & 0.866 & 0.748 & 0.056 & 0.169 & 0.683 & 0.560 \\
\hline DT & 0.300 & 0.692 & 0.870 & 0.744 & 75.862 & 63.636 & 84.482 & 84.058 & 0.570 & 0.667 & 0.862 & 0.829 & 0.154 & 0.248 & 0.683 & 0.632 \\
\hline $\mathbf{R F}$ & 0.235 & 0.571 & 0.896 & 0.563 & 77.586 & 59.090 & 87.931 & 79.710 & 0.807 & 0.714 & 0.948 & 0.838 & 0.111 & 0.245 & 0.753 & 0.548 \\
\hline
\end{tabular}


Table 8. MC1 Results

\begin{tabular}{|c|c|c|c|c|c|c|c|c|c|c|c|c|c|c|c|c|}
\hline \multirow{2}{*}{ Classifiers } & \multicolumn{4}{|c|}{ F-Measure } & \multicolumn{4}{|c|}{ Accuracy } & \multicolumn{4}{|c|}{ ROC Area } & \multicolumn{4}{|c|}{ MCC } \\
\hline & No Tec & RUS & ROS & SMOTE & No Tec & RUS & ROS & SMOTE & $\begin{array}{l}\text { No } \\
\text { Tec }\end{array}$ & RUS & ROS & SMOTE & $\begin{array}{l}\text { No } \\
\text { Tec }\end{array}$ & RUS & ROS & SMOTE \\
\hline NB & 0.217 & 0.353 & 0.505 & 0.211 & 93.856 & 50.000 & 63.139 & 87.416 & 0.826 & 0.590 & 0.797 & 0.778 & 0.208 & 0.153 & 0.330 & 0.204 \\
\hline MLP & ? & 0.727 & 0.887 & 0.400 & 97.610 & 72.727 & 88.737 & 96.476 & 0.805 & 0.897 & 0.970 & 0.829 & $?$ & 0.504 & 0.776 & 0.391 \\
\hline RBF & $?$ & 0.727 & 0.781 & ? & 97.610 & 72.727 & 77.815 & 96.476 & 0.781 & 0.889 & 0.889 & 0.764 & $?$ & 0.504 & 0.557 & ? \\
\hline SVM & ? & 0.700 & 0.821 & ? & 97.610 & 72.727 & 81.570 & 96.476 & 0.500 & 0.769 & 0.815 & 0500 & $?$ & 0.568 & 0.631 & ? \\
\hline kNN & 0.333 & 0.696 & 0.995 & 0.585 & 97.269 & 68.181 & 99.488 & 97.147 & 0.638 & 0.697 & 0.995 & 0.779 & 0.325 & 0.388 & 0.990 & 0.571 \\
\hline kStar & 0.182 & 0.667 & 0.998 & 0.514 & 96.928 & 68.181 & 99.829 & 97.147 & 0.631 & 0.701 & 1.000 & 0.856 & 0.174 & 0.437 & 0.997 & 0.511 \\
\hline OneR & 0.200 & 0.421 & 0.963 & 0.240 & 97.269 & 50.000 & 96.075 & 96.811 & 0.568 & 0.543 & 0.960 & 0.571 & 0.206 & 0.094 & 0.924 & 0.319 \\
\hline PART & 0.333 & 0.727 & 0.990 & 0.500 & 97.269 & 72.727 & 98.976 & 96.979 & 0.684 & 0.658 & 0.988 & 0.890 & 0.325 & 0.504 & 0.980 & 0.492 \\
\hline DT & ? & 0.667 & 0.984 & 0.474 & 97.610 & 68.181 & 98.293 & 96.644 & 0.500 & 0.765 & 0.982 & 0.750 & $?$ & 0.437 & 0.966 & 0.459 \\
\hline RF & 0.000 & 0.526 & 0.998 & 0.385 & 97.440 & 59.090 & 99.829 & 97.315 & 0.864 & 0.769 & 1.000 & 0.973 & -0.006 & 0.302 & 0.997 & 0.481 \\
\hline
\end{tabular}

Table 9. MC2 Results

\begin{tabular}{|c|c|c|c|c|c|c|c|c|c|c|c|c|c|c|c|c|}
\hline \multirow{2}{*}{ Classifiers } & \multicolumn{4}{|c|}{ F-Measure } & \multicolumn{4}{|c|}{ Accuracy } & \multicolumn{4}{|c|}{ ROC Area } & \multicolumn{4}{|c|}{ MCC } \\
\hline & No Tec & RUS & ROS & SMOTE & No Tec & RUS & ROS & SMOTE & $\begin{array}{l}\text { No } \\
\text { Tec }\end{array}$ & RUS & ROS & SMOTE & $\begin{array}{l}\text { No } \\
\text { Tec }\end{array}$ & RUS & ROS & SMOTE \\
\hline NB & 0.526 & 0.600 & 0.522 & 0.596 & 75.675 & 69.230 & 70.270 & 62.000 & 0.795 & 0.726 & 0.847 & 0.735 & 0.444 & 0.386 & 0.477 & 0.301 \\
\hline MLP & 0.519 & 0.300 & 0.750 & 0.654 & 64.864 & 46.153 & 78.378 & 64.000 & 0.753 & 0.507 & 0.865 & 0.785 & 0.243 & -0.11 & 0.564 & 0.298 \\
\hline RBF & 0.444 & 0.538 & 0.667 & 0.750 & 72.973 & 53.846 & 70.270 & 72.000 & 0.766 & 0.639 & 0.824 & 0.829 & 0.371 & 0.264 & 0.399 & 0.434 \\
\hline SVM & 0.222 & 0.583 & 0.621 & 0.720 & 62.162 & 61.538 & 70.270 & 72.000 & 0.514 & 0.688 & 0.690 & 0.739 & 0.04 & 0.356 & 0.404 & 0.478 \\
\hline kNN & 0.545 & 0.522 & 0.839 & 0.800 & 72.973 & 57.692 & 86.486 & 76.000 & 0.668 & 0.625 & 0.822 & 0.747 & 0.374 & 0.234 & 0.734 & 0.503 \\
\hline kStar & 0.348 & 0.400 & 0.765 & 0.862 & 59.459 & 65.384 & 78.378 & 84.000 & 0.510 & 0.576 & 0.838 & 0.816 & 0.062 & 0.159 & 0.565 & 0.672 \\
\hline OneR & & 0.385 & 0.647 & 0.807 & 64.864 & 38.461 & 67.567 & 78.000 & 0.553 & 0.451 & 0.674 & 0.778 & 0.137 & -0.09 & 0.347 & 0.552 \\
\hline PART & 0.667 & 0.500 & 0.727 & 0.727 & 78.378 & 53.846 & 75.675 & 70.000 & 0.724 & 0.639 & 0.768 & 0.755 & 0.512 & 0.184 & 0.509 & 0.399 \\
\hline DT & 0.435 & 0.522 & 0.629 & 0.721 & 64.864 & 57.692 & 64.864 & 66.000 & 0.615 & 0.639 & 0.715 & 0.631 & 0.189 & 0.234 & 0.296 & 0.290 \\
\hline $\mathbf{R F}$ & 0.480 & 0.435 & 0.774 & 0.847 & 64.864 & 50.000 & 81.081 & 82.000 & 0.646 & 0.556 & 0.937 & 0.878 & 0.216 & 0.065 & 0.623 & 0.629 \\
\hline
\end{tabular}

Table 10. MW1 Results

\begin{tabular}{|c|c|c|c|c|c|c|c|c|c|c|c|c|c|c|c|c|}
\hline \multirow{2}{*}{ Classifiers } & \multicolumn{4}{|c|}{ F-Measure } & \multicolumn{4}{|c|}{ Accuracy } & \multicolumn{4}{|c|}{ ROC Area } & \multicolumn{4}{|c|}{ MCC } \\
\hline & No Tec & RUS & ROS & SMOTE & No Tec & RUS & ROS & SMOTE & $\begin{array}{l}\text { No } \\
\text { Tec }\end{array}$ & RUS & ROS & SMOTE & $\begin{array}{l}\text { No } \\
\text { Tec } \\
\end{array}$ & RUS & ROS & SMOTE \\
\hline NB & 0.435 & 0.706 & 0.740 & 0.516 & 82.666 & 66.666 & 74.666 & 81.707 & 0.791 & 0.630 & 0.793 & 0.842 & 0.367 & 0.327 & 0.506 & 0.427 \\
\hline MLP & 632 & 0.706 & 0.930 & 0.522 & 90.666 & 66.666 & 92.000 & 86.585 & 0.843 & 0.722 & 0.938 & 0.790 & 0.589 & 0.327 & 0.849 & 0.444 \\
\hline RBF & $?$ & 0.667 & 0.819 & 0.400 & 89.333 & 66.666 & 80.000 & 85.365 & 0.808 & 0.778 & 0.828 & 0.829 & ? & 0.389 & 0.598 & 0.329 \\
\hline SVM & $?$ & 0.667 & 0.800 & 0.500 & 89.333 & 66.666 & 80.000 & 87.804 & 0.500 & 0.694 & 0.804 & 0.687 & $?$ & 0.389 & 0.607 & 0.445 \\
\hline kNN & 444 & 0.706 & 0.909 & 0.538 & 86.666 & 66.666 & 89.333 & 85.365 & 0.705 & 0.667 & 0.854 & 0.742 & 0.373 & 0.327 & 0.802 & 0.454 \\
\hline kStar & 0.133 & 0.800 & 0.909 & 0.552 & 82.666 & 80.000 & 89.333 & 84.146 & 0.543 & 0.778 & 0.967 & 0.860 & 0.038 & 0.667 & 0.802 & 0.469 \\
\hline OneR & 0.200 & 0.667 & 0.833 & 0.333 & 89.333 & 66.666 & 81.333 & 85.365 & 0.555 & 0.694 & 0.809 & 0.604 & 0.211 & 0.389 & 0.626 & 0.281 \\
\hline PART & 0.167 & 0.706 & 0.930 & 0.522 & 86.666 & 66.666 & 92.000 & 86.585 & 0.314 & 0.630 & 0.940 & 0.656 & 0.110 & 0.327 & 0.849 & 0.444 \\
\hline DT & 0.167 & 0.750 & 0.920 & 0.417 & 86.666 & 73.333 & 90.666 & 82.926 & 0.314 & 0.722 & 0.900 & 0.740 & 0.110 & 0.491 & 0.825 & 0.317 \\
\hline RF & 0.182 & 0.667 & 0.952 & 0.609 & 88.000 & 66.666 & 94.666 & 89.024 & 0.766 & 0.741 & 1.000 & 0.896 & 0.150 & 0.389 & 0.897 & 0.546 \\
\hline
\end{tabular}


Table 11. PC1 Results

\begin{tabular}{|c|c|c|c|c|c|c|c|c|c|c|c|c|c|c|c|c|}
\hline \multirow{2}{*}{ Classifiers } & \multicolumn{4}{|c|}{ F-Measure } & \multicolumn{4}{|c|}{ Accuracy } & \multicolumn{4}{|c|}{ ROC Area } & \multicolumn{4}{|c|}{ MCC } \\
\hline & No Tec & RUS & ROS & SMOTE & No Tec & RUS & ROS & SMOTE & $\begin{array}{l}\text { No } \\
\text { Tec }\end{array}$ & RUS & ROS & SMOTE & $\begin{array}{l}\text { No } \\
\text { Tec }\end{array}$ & RUS & ROS & SMOTE \\
\hline NB & 0.400 & 0.583 & 0.530 & 0.485 & 89.705 & 69.697 & 65.024 & 84.545 & 0.879 & 0.818 & 0.846 & 0.842 & 0.400 & 0.442 & 0.382 & 0.403 \\
\hline MLP & 0.462 & 0.500 & 0.929 & 0.508 & 96.568 & 57.575 & 92.118 & 85.909 & 0.779 & 0.713 & 0.942 & 0.910 & 0.538 & 0.149 & 0.852 & 0.443 \\
\hline RBF & 0.154 & 0.667 & 0.851 & 0.130 & 94.607 & 66.666 & 83.743 & 81.818 & 0.875 & 0.790 & 0.901 & 0.870 & 0.161 & 0.335 & 0.677 & 0.104 \\
\hline SVM & ? & 0.688 & 0.853 & 0.000 & 95.098 & 69.697 & 83.743 & 82.272 & 0.5 & 0.697 & 0.835 & 0.497 & ? & 0.393 & 0.680 & -0.031 \\
\hline kNN & 0.286 & 0.774 & 0.963 & 0.507 & 92.647 & 78.787 & 96.059 & 85.000 & 0.629 & 0.787 & 0.979 & 0.691 & 0.247 & 0.576 & 0.924 & 0.426 \\
\hline kStar & 0.176 & 0.552 & 0.955 & 0.667 & 86.274 & 60.606 & 95.073 & 88.636 & 0.673 & 0.728 & 0.983 & 0.920 & 0.128 & 0.211 & 0.905 & 0.598 \\
\hline OneR & 0.154 & 0.737 & 0.860 & 0.276 & 94.607 & 69.697 & 84.729 & 80.909 & 0.545 & 0.702 & 0.845 & 0.572 & 0.161 & 0.429 & 0.697 & 0.190 \\
\hline PART & 0.462 & 0.667 & 0.963 & 0.514 & 93.137 & 69.697 & 96.059 & 84.545 & 0.889 & 0.691 & 0.967 & 0.771 & 0.440 & 0.394 & 0.922 & 0.425 \\
\hline DT & 0.500 & 0.750 & 0.940 & 0.606 & 93.137 & 75.757 & 93.596 & 88.181 & 0.718 & 0.721 & 0.948 & 0.808 & 0.490 & 0.515 & 0.874 & 0.547 \\
\hline RF & 0.429 & 0.778 & 0.946 & 0.610 & 96.078 & 75.757 & 94.088 & 89.545 & 0.858 & 0.860 & 0.999 & 0.941 & 0.459 & 0.534 & 0.887 & 0.588 \\
\hline
\end{tabular}

Table 12. PC2 Results

\begin{tabular}{|c|c|c|c|c|c|c|c|c|c|c|c|c|c|c|c|c|}
\hline \multirow[b]{2}{*}{ Classifiers } & \multicolumn{4}{|c|}{ F-Measure } & \multicolumn{4}{|c|}{ Accuracy } & \multicolumn{4}{|c|}{ ROC Area } & \multicolumn{4}{|c|}{ MCC } \\
\hline & No Tec & RUS & ROS & SMOTE & No Tec & RUS & ROS & SMOTE & $\begin{array}{l}\text { No } \\
\text { Tec }\end{array}$ & RUS & ROS & SMOTE & $\begin{array}{l}\text { No } \\
\text { Tec }\end{array}$ & RUS & ROS & SMOTE \\
\hline NB & 0.000 & 0.667 & 0.752 & 0.250 & 94.470 & 70.000 & 76.958 & 91.855 & 0.751 & 0.875 & 0.826 & 0.844 & -0.028 & 0.535 & 0.542 & 0.207 \\
\hline MLP & 0.000 & 0.923 & 0.955 & 0.273 & 96.774 & 90.000 & 95.391 & 92.760 & 0.746 & 0.958 & 0.939 & 0.866 & -0.015 & 0.802 & 0.912 & 0.236 \\
\hline RBF & $?$ & 0.923 & 0.800 & ? & 97.695 & 90.000 & 78.801 & 94.570 & 0.724 & 0.958 & 0.885 & 0.812 & $?$ & 0.802 & 0.583 & ? \\
\hline SVM & $?$ & 0.667 & 0.790 & $?$ & 97.695 & 70.000 & 77.419 & 94.570 & 0.500 & 0.750 & 0.775 & 0.500 & $?$ & 0.535 & 0.558 & $?$ \\
\hline kNN & 0.000 & 0.833 & 0.955 & 0.400 & 96.774 & 80.000 & 95.391 & 94.570 & 0.495 & 0.792 & 0.941 & 0.657 & -0.015 & 0.583 & 0.912 & 0.381 \\
\hline kStar & 0.167 & 0.833 & 0.973 & 0.182 & 95.391 & 80.000 & 97.235 & 91.855 & 0.791 & 0.813 & 1.000 & 0.696 & 0.146 & 0.583 & 0.946 & 0.140 \\
\hline OneR & 0.000 & 0.923 & 0.930 & 0.375 & 97.235 & 90.000 & 92.626 & 95.475 & 0.498 & 0.875 & 0.927 & 0.623 & -0.01 & 0.802 & 0.862 & 0.417 \\
\hline PART & 0.000 & 0.833 & 0.968 & $?$ & 96.774 & 80.000 & 96.774 & 94.570 & 0.623 & 0.854 & 0.979 & 0.871 & -0.015 & 0.583 & 0.937 & $?$ \\
\hline DT & $?$ & 0.833 & 0.973 & 0.250 & 97.695 & 80.000 & 97.235 & 94.570 & 0.579 & 0.854 & 0.977 & 0.813 & $?$ & 0.583 & 0.946 & 0.267 \\
\hline $\mathbf{R F}$ & $?$ & 0.923 & 0.977 & $?$ & 97.695 & 90.000 & 97.695 & 94.570 & 0.731 & 0.958 & 1.000 & 0.968 & $?$ & 0.802 & 0.955 & $?$ \\
\hline
\end{tabular}

Table 13. PC3 Results

\begin{tabular}{|c|c|c|c|c|c|c|c|c|c|c|c|c|c|c|c|c|}
\hline \multirow{2}{*}{ Classifiers } & \multicolumn{4}{|c|}{ F-Measure } & \multicolumn{4}{|c|}{ Accuracy } & \multicolumn{4}{|c|}{ ROC Area } & \multicolumn{4}{|c|}{ MCC } \\
\hline & No Tec & RUS & ROS & SMOTE & No Tec & RUS & ROS & SMOTE & $\begin{array}{l}\text { No } \\
\text { Tec }\end{array}$ & RUS & ROS & SMOTE & $\begin{array}{l}\text { No } \\
\text { Tec }\end{array}$ & RUS & ROS & SMOTE \\
\hline NB & 0.257 & 0.754 & 0.634 & 0.576 & 28.797 & 78.205 & 49.683 & 75.493 & 0.773 & 0.823 & 0.722 & 0.832 & 0.088 & 0.561 & 0.013 & 0.452 \\
\hline MLP & 0.261 & 0.694 & 0.819 & 0.482 & 83.860 & 71.794 & 81.012 & 79.436 & 0.796 & 0.751 & 0.859 & 0.848 & 0.183 & 0.433 & 0.627 & 0.356 \\
\hline RBF & ? & 0.763 & 0.764 & 0.274 & 86.392 & 76.923 & 75.316 & 76.056 & 0.795 & 0.798 & 0.807 & 0.803 & $?$ & 0.542 & 0.511 & 0.152 \\
\hline SVM & $?$ & 0.763 & 0.731 & 0.136 & 86.320 & 76.923 & 71.835 & 78.591 & 0.500 & 0.772 & 0.720 & 0.528 & $?$ & 0.542 & 0.442 & 0.120 \\
\hline kNN & 0.353 & 0.667 & 0.928 & 0.535 & 86.075 & 70.512 & 92.721 & 81.408 & 0.616 & 0.700 & 0.919 & 0.702 & 0.294 & 0.404 & 0.856 & 0.421 \\
\hline kStar & 0.267 & 0.667 & 0.904 & 0.604 & 82.594 & 70.512 & 89.873 & 82.253 & 0.749 & 0.702 & 0.962 & 0.870 & 0.173 & 0.404 & 0.806 & 0.491 \\
\hline OneR & 0.226 & 0.747 & 0.774 & 0.462 & 87.025 & 75.641 & 75.949 & 84.225 & 0.562 & 0.758 & 0.761 & 0.051 & 0.245 & 0.514 & 0.527 & 0.450 \\
\hline PART & ? & 0.658 & 0.906 & 0.417 & 86.392 & 65.384 & 90.506 & 81.126 & 0.790 & 0.696 & 0.929 & 0.747 & ? & 0.318 & 0.811 & 0.339 \\
\hline DT & 0.358 & 0.718 & 0.896 & 0.592 & 86.392 & 71.794 & 89.240 & 83.662 & 0.664 & 0.742 & 0.908 & 0.711 & 0.304 & 0.444 & 0.789 & 0.491 \\
\hline $\mathbf{R F}$ & 0.226 & 0.767 & 0.911 & 0.641 & 87.025 & 78.205 & 90.822 & 86.760 & 0.855 & 0.796 & 0.983 & 0.900 & 0.245 & 0.563 & 0.822 & 0.571 \\
\hline
\end{tabular}


Table 14. PC4 Results

\begin{tabular}{|c|c|c|c|c|c|c|c|c|c|c|c|c|c|c|c|c|}
\hline \multirow[b]{2}{*}{ Classifiers } & \multicolumn{4}{|c|}{ F-Measure } & \multicolumn{4}{|c|}{ Accuracy } & \multicolumn{4}{|c|}{ ROC Area } & \multicolumn{4}{|c|}{ MCC } \\
\hline & No Tec & RUS & ROS & SMOTE & No Tec & RUS & ROS & SMOTE & $\begin{array}{l}\text { No } \\
\text { Tec }\end{array}$ & RUS & ROS & SMOTE & $\begin{array}{l}\text { No } \\
\text { Tec }\end{array}$ & RUS & ROS & SMOTE \\
\hline NB & 0.404 & 0.617 & 0.650 & 0.477 & 86.0892 & 70.754 & 69.816 & 79.262 & 0.807 & 0.766 & 0.787 & 0.844 & 0.334 & 0.456 & 0.436 & 0.396 \\
\hline MLP & 0.562 & 0.739 & 0.899 & 0.736 & 89.7638 & 72.641 & 89.238 & 85.483 & 0.898 & 0.814 & 0.938 & 0.918 & 0.515 & 0.458 & 0.784 & 0.638 \\
\hline RBF & 0.250 & 0.717 & 0.788 & 0.476 & 87.4016 & 71.698 & 77.690 & 80.184 & 0.862 & 0.824 & 0.887 & 0.887 & 0.279 & 0.434 & 0.553 & 0.424 \\
\hline SVM & 0.286 & 0.754 & 0.805 & 0.561 & 88.189 & 73.584 & 78.477 & 83.410 & 0.583 & 0.738 & 0.782 & 0.696 & 0.342 & 0.482 & 0.569 & 0.539 \\
\hline kNN & 0.438 & 0.738 & 0.954 & 0.679 & 85.8268 & 69.811 & 95.013 & 83.871 & 0.667 & 0.701 & 0.945 & 0.778 & 0.359 & 0.425 & 0.902 & 0.573 \\
\hline kStar & 0.330 & 0.683 & 0.917 & 0.733 & 81.8898 & 62.264 & 90.551 & 85.253 & 0.734 & 0.669 & 0.985 & 0.905 & 0.225 & 0.275 & 0.821 & 0.633 \\
\hline OneR & 0.361 & 0.786 & 0.841 & 0.620 & 87.9265 & 77.358 & 82.677 & 85.023 & 0.614 & 0.775 & 0.825 & 0.726 & 0.352 & 0.555 & 0.653 & 0.589 \\
\hline PART & 0.481 & 0.828 & 0.916 & 0.785 & 85.3018 & 81.132 & 90.813 & 87.788 & 0.776 & 0.801 & 0.923 & 0.892 & 0.396 & 0.641 & 0.818 & 0.705 \\
\hline DT & 0.583 & 0.810 & 0.936 & 0.742 & 86.8766 & 79.245 & 92.913 & 86.405 & 0.834 & 0.775 & 0.944 & 0.863 & 0.514 & 0.602 & 0.862 & 0.650 \\
\hline RF & 0.532 & 0.831 & 0.947 & 0.823 & 90.2887 & 81.132 & 94.225 & 91.474 & 0.945 & 0.877 & 0.995 & 0.964 & 0.516 & 0.647 & 0.888 & 0.773 \\
\hline
\end{tabular}

Table 15. PC5 Results

\begin{tabular}{|c|c|c|c|c|c|c|c|c|c|c|c|c|c|c|c|c|}
\hline \multirow{2}{*}{ Classifiers } & \multicolumn{4}{|c|}{ F-Measure } & \multicolumn{4}{|c|}{ Accuracy } & \multicolumn{4}{|c|}{ ROC Area } & \multicolumn{4}{|c|}{ MCC } \\
\hline & No Tec & RUS & ROS & SMOTE & No Tec & RUS & ROS & SMOTE & $\begin{array}{l}\text { No } \\
\text { Tec }\end{array}$ & RUS & ROS & SMOTE & $\begin{array}{l}\text { No } \\
\text { Tec }\end{array}$ & RUS & ROS & SMOTE \\
\hline NB & 0.269 & 0.281 & 0.333 & 0.385 & $|75.3937|$ & 55.272 & 56.692 & 64.860 & 0.725 & 0.695 & 0.732 & 0.748 & 0.245 & 0.167 & 0.214 & 0.282 \\
\hline MLP & 0.299 & 0.702 & 0.738 & 0.663 & 74.2126 & 67.636 & 73.622 & 69.195 & 0.751 & 0.698 & 0.797 & 0.771 & 0.216 & 0.357 & 0.473 & 0.384 \\
\hline RBF & 0.235 & 0.699 & 0.686 & 0.662 & 75.5906 & 68.727 & 68.110 & 72.291 & 0.732 & 0.719 & 0.751 & 0.788 & 0.251 & 0.375 & 0.362 & 0.429 \\
\hline SVM & 0.097 & 0.671 & 0.710 & 0.612 & \begin{tabular}{|l|l|}
74.2126 \\
\end{tabular} & 65.090 & 68.897 & 68.421 & 0.524 & 0.651 & 0.688 & 0.671 & 0.173 & 0.304 & 0.378 & 0.349 \\
\hline kNN & 0.498 & 0.671 & 0.858 & 0.702 & 73.0315 & 66.909 & 85.039 & 74.767 & 0.657 & 0.669 & 0.857 & 0.741 & 0.314 & 0.338 & 0.702 & 0.483 \\
\hline kStar & 0.431 & 0.680 & 0.849 & 0.731 & 69.8819 & 66.181 & 83.661 & 75.541 & 0.629 & 0.697 & 0.903 & 0.818 & 0.227 & 0.325 & 0.678 & 0.511 \\
\hline OneR & 0.387 & 0.611 & 0.718 & 0.662 & 71.2598 & 62.909 & 70.866 & 76.470 & 0.594 & 0.629 & 0.708 & 0.736 & 0.209 & 0.260 & 0.417 & 0.528 \\
\hline PART & 0.335 & 0.733 & 0.766 & 0.669 & 75.7874 & 67.636 & 71.060 & 75.387 & 0.739 & 0.744 & 0.809 & 0.792 & 0.274 & 0.387 & 0.464 & 0.495 \\
\hline DT & 0.531 & 0.695 & 0.824 & 0.737 & 75.000 & 65.454 & 81.692 & 76.935 & 0.703 & 0.626 & 0.819 & 0.761 & 0.361 & 0.319 & 0.634 & 0.532 \\
\hline RF & 0.450 & 0.716 & 0.877 & 0.785 & 75.9843 & 69.090 & 87.204 & 81.114 & 0.805 & 0.766 & 0.953 & 0.897 & 0.322 & 0.387 & 0.744 & 0.617 \\
\hline
\end{tabular}

The classification results after implementing each of the resampling techniques (as mentioned in the comparison framework) on all of the used datasets are reflected in the tables (from Table. 4 to Table. 15). The sub column named "No Tec" (no technique of class balancing is used) in each of the accuracy measure refers to the published results from [7], where same classifiers and datasets are used without any resampling technique. The purpose of using those results in this research is to compare the effectiveness of resampling techniques. From the results it has been observed that overall in each performance measure, ROS performed better in all datasets with most of the classifiers. However in Accuracy, besides the ROS, most of the classifiers also performed better when no sampling technique was used. RUS did not perform well except in $\mathrm{KC} 1$ dataset with few of the classifiers. SMOTE on the other hand performed better in $\mathrm{MC} 2$ dataset with most of the classifiers. The class imbalance issue reflected by [7] in most of the datasets are resolved by the used resampling techniques with one exceptional case of PC2 dataset in which this issue still exists as shown in Table 12.

\section{CONCLUSION}

The performance of supervised machine learning classifiers can be biased due to class imbalance issue in the datasets. This research analyzed the performance of three widely used resampling techniques on class imbalance issue during software defect prediction. The used resampling techniques are: Random Under Sampling, Random Over Sampling and Synthetic Minority Oversampling Technique (SMOTE). Twelve cleaned publically available NASA datasets are used for experiments along with 10 widely used classifiers including: Naïve Bayes (NB), Multi-Layer Perceptron (MLP). Radial Basis Function (RBF), Support Vector Machine (SVM), K Nearest Neighbor (KNN), kStar (K*), One Rule (OneR), PART, Decision Tree (DT), and Random Forest (RF). The performance is measured in terms of F-measure, Accuracy, MCC and ROC. This paper compared the performance of resampling techniques with the results of a published research in which no resampling technique is used however classifiers and datasets are the same. According to results, 
Random Over Sampling outperformed other techniques with most of the classifiers in all datasets. The resampling techniques resolved the issue of class imbalance in 11 out of 12 datasets with the exception of one dataset named PC2. It is suggested for future that ensemble classifiers should be used along with resampling techniques to further improve the performance.

\section{REFERENCES}

[1] U. R. Salunkhe and S. N. Mali, "A hybrid approach for class imbalance problem in customer churn prediction: A novel extension to under-sampling," Int. J. Intell. Syst. Appl., vol. 10, no. 5, pp. 71-81, 2018.

[2] S. Wang and X. Yao, "Using class imbalance learning for software defect prediction," IEEE Trans. Reliab., vol. 62, no. 2, pp. 434-443, 2013.

[3] X. Yu, J. Liu, Z. Yang, X. Jia, Q. Ling, and S. Ye, "Learning from Imbalanced Data for Predicting the Number of Software Defects," Proc. - Int. Symp. Softw. Reliab. Eng. ISSRE, vol. 2017-October, pp. 78-89, 2017.

[4] L. Pelayo and S. Dick, "Applying novel resampling strategies to software defect prediction," Annu. Conf. North Am. Fuzzy Inf. Process. Soc. - NAFIPS, pp. 69-72, 2007.

[5] D. Tomar and S. Agarwal, "Prediction of Defective Software Modules Using Class Imbalance Learning," Appl. Comput. Intell. Soft Comput., vol. 2016, pp. 1-12, 2016.

[6] N. F. Hordri, S. S. Yuhaniz, N. F. M. Azmi, and S. M. Shamsuddin, "Handling class imbalance in credit card fraud using resampling methods," Int. J. Adv. Comput. Sci. Appl., vol. 9, no. 11, pp. 390-396, 2018.

[7] A. Iqbal, S. Aftab, U. Ali, Z. Nawaz, L. Sana, M. Ahmad, and A. Husen "Performance Analysis of Machine Learning Techniques on Software Defect Prediction using NASA Datasets," Int. J. Adv. Comput. Sci. Appl., vol. 10, no. $5,2019$.

[8] D. Bowes, T. Hall, and J. Petrić, "Software defect prediction: do different classifiers find the same defects?," Softw. Qual. J., vol. 26, no. 2, pp. 525-552, 2018

[9] A. Iqbal, S. Aftab, I. Ullah, M. S. Bashir, and M. A. Saeed, "A Feature Selection based Ensemble Classification Framework for Software Defect Prediction," Int. J. Mod. Educ. Comput. Sci., vol. 11, no. 9, pp. 54-64, 2019.

[10] M. Ahmad, S. Aftab, I. Ali, and N. Hameed, "Hybrid Tools and Techniques for Sentiment Analysis: A Review," Int. J. Multidiscip. Sci. Eng., vol. 8, no. 3, 2017.

[11] M. Ahmad, S. Aftab, S. S. Muhammad, and S. Ahmad, "Machine Learning Techniques for Sentiment Analysis: A Review," Int. J. Multidiscip. Sci. Eng., vol. 8, no. 3, p. 27, 2017.

[12] M. Ahmad, S. Aftab, M. S. Bashir, N. Hameed, I. Ali, and Z. Nawaz, "SVM Optimization for Sentiment Analysis," Int. J. Adv. Comput. Sci. Appl., vol. 9, no. 4, 2018.

[13] M. Ahmad, S. Aftab, M. S. Bashir, and N. Hameed, "Sentiment Analysis using SVM: A Systematic Literature Review," Int. J. Adv. Comput. Sci. Appl., vol. 9, no. 2, 2018.

[14] M. Ahmad, S. Aftab, and I. Ali, "Sentiment Analysis of Tweets using SVM," Int. J. Comput. Appl., vol. 177, no. 5, pp. 25-29, 2017.

[15] M. Ahmad and S. Aftab, "Analyzing the Performance of SVM for Polarity Detection with Different Datasets," Int.
J. Mod. Educ. Comput. Sci., vol. 9, no. 10, pp. 29-36, 2017.

[16] S. Aftab, M. Ahmad, N. Hameed, M. S. Bashir, I. Ali, and Z. Nawaz, "Rainfall Prediction in Lahore City using Data Mining Techniques,” Int. J. Adv. Comput. Sci. Appl., vol. 9, no. 4, 2018.

[17] S. Aftab, M. Ahmad, N. Hameed, M. S. Bashir, I. Ali, and Z. Nawaz, "Rainfall Prediction using Data Mining Techniques: A Systematic Literature Review,” Int. J. Adv. Comput. Sci. Appl., vol. 9, no. 5, 2018.

[18] A. Iqbal and S. Aftab, "A Feed-Forward and Pattern Recognition ANN Model for Network Intrusion Detection," Int. J. Comput. Netw. Inf. Secur., vol. 11, no. 4, pp. 19-25, 2019.

[19] A. Iqbal, S. Aftab, I. Ullah, M. A. Saeed, and A. Husen, "A Classification Framework to Detect DoS Attacks," Int. J. Comput. Netw. Inf. Secur., vol. 11, no. 9, pp. 40-47, 2019.

[20] S. Huda et al., "A Framework for Software Defect Prediction and Metric Selection," IEEE Access, vol. 6, no. c, pp. 2844-2858, 2017.

[21] E. Erturk and E. Akcapinar, "A comparison of some soft computing methods for software fault prediction," Expert Syst. Appl., vol. 42, no. 4, pp. 1872-1879, 2015.

[22] M. Shepperd, Q. Song, Z. Sun and C. Mair, "Data Quality: Some Comments on the NASA Software Defect Datasets," IEEE Trans. Softw. Eng., vol. 39, pp. 1208-1215, 2013.

[23] "NASA Defect Dataset." [Online]. Available: https://github.com/klainfo/NASADefectDataset. [Accessed: 14-August-2019].

[24] B. Ghotra, S. McIntosh, and A. E. Hassan, "Revisiting the impact of classification techniques on the performance of defect prediction models," Proc. - Int. Conf. Softw. Eng., vol. 1, pp. 789-800, 2015

[25] G. Czibula, Z. Marian, and I. G. Czibula, "Software defect prediction using relational association rule mining," Inf. Sci. (Ny)., vol. 264, pp. 260-278, 2014.

[26] D. Rodriguez, I. Herraiz, R. Harrison, J. Dolado, and J. C. Riquelme, "Preliminary comparison of techniques for dealing with imbalance in software defect prediction," in Proceedings of the 18th International Conference on Evaluation and Assessment in Software Engineering. ACM, p. 43, 2014.

\section{Authors' Profiles}

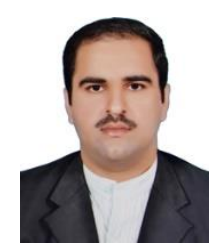

Ahmed Iqbal is the student of MS Computer Science with the specialization of Software Engineering in Virtual University of Pakistan. $\mathrm{He}$ received the degree, Master of Information Technology (MIT) from Virtual University of Pakistan in 2016. His research interest includes Software Engineering and

Data Mining.

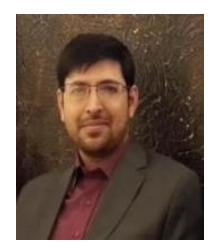

Shabib Aftab received MS Degree in Computer Science from COMSATS Institute of Information Technology Lahore, Pakistan, and M.Sc degree in Information Technology from Punjab University College of Information Technology (PUCIT) Lahore, Pakistan. Currently he is serving as Lecturer Computer Sciences at Virtual University of Pakistan. His 
research areas include Data Mining and Software Process Improvement.

Faseeha Matloob is student of MS Computer Science with the specialization of Software Engineering in Virtual University of Pakistan. Her research interest includes Software Engineering and Data Mining.

How to cite this paper: Ahmed Iqbal, Shabib Aftab, Faseeha Matloob, "Performance Analysis of Resampling Techniques on Class Imbalance Issue in Software Defect Prediction", International Journal of Information Technology and Computer Science(IJITCS), Vol.11, No.11, pp.44-53, 2019. DOI: 10.5815/ijitcs.2019.11.05 